\title{
¿Refugiados de primera o segunda clase?
}

\author{
Andrea Borja Gonzalo*
}

\section{Resumen}

El artículo propuesto busca examinar los acuerdos, medidas, programas y planes de acción especiales que la Comisión Europea ha puesto en marcha para abordar la situación migratoria que se vive en Europa, y cómo estos discriminan a las personas solicitantes de asilo en función de su nacionalidad. Grecia junto con Italia, son los primeros países de la Unión Europea a los que llegan las personas migrantes/refugiadas/solicitantes de asilo y donde estas experimentan por primera vez, dentro de las fronteras europeas, las consecuencias directas de las decisiones, acuerdos y medidas implementadas. El presente artículo se centrará en el caso griego. La mayoría de las investigaciones actuales sobre desplazamientos forzados se centran en el conflicto sirio, olvidándose del resto de conflictos y personas que buscan refugio en Europa provenientes de países como Afganistán, Irak, Irán, Pakistán y Palestina, entre otros. Multitud de personas solicitantes de asilo llegan a Grecia cada mes desde hace más de dos años. El acceso o restricción a servicios y programas dependiendo de la nacionalidad del o la solicitante de asilo supone una clara discriminación por origen nacional y la aparición de categorías como refugiados de primera o segunda clase. El programa de reubicación es un claro ejemplo, ya que únicamente siete nacionalidades, además de las personas apátridas, pueden acogerse a dicho programa.

\section{Palabras clave}

Asilo; Unión Europea; Migración; Discriminación; Protección internacional; Acuerdos; Tercer país seguro.

\section{TITLE}

First or second class refugees?

\section{Abstract}

The article seeks to analyze the special agreements, measures, programs and action plans the European Commission has implemented to address the current migratory situation in Europe and how these mechanisms discriminate against asylum seekers depending on their nationality. Together, Greece and Italy are the countries in the European Union where most migrants / refugees and asylum seekers first arrive and experience the direct consequences of these agreements, measures and decisions taken within European borders. With that in mind, this article will focus on the Greek case. The most recent research projects on forced migration focus on the Syrian conflict, paying little attention to other conflicts and nationalities such as the Afghan, Iraqi, Iranian, Pakistani and Palestinian nationals seeking refuge in Europe. Significant numbers of asylum seekers from the above mentioned countries have been arriving in Greece every month since 2015. Access or restriction to services and programs depending on the nationality of the asylum seeker implies a clear discrimination which is based on their national origin, as well as the emergence of categories like first or second class refugee. The relocation program clearly discriminates against asylum applicants depending on their country of origin since just 7 different nationalities, as well as those considered stateless persons, are eligible for such program.

\section{KEYWORDS}

Asylum; European Union; Migration; Discrimination; International protection; Agreements; Safe third country.

\section{*Andrea} BORJA GONZALO, Estudiante del programa de doctorado en Relaciones de Género y Estudios Feministas de la Universidad de Zaragoza. La autora trabaja desde 2015 para una red creada por mujeres migrantes y refugiadas en Grecia.

\section{Recibido:}

2017-04-22

Aceptado:

2017-09-20

DOI: $10.15366 /$

relacionesinternacio nales2017.36.002 


\section{ntroducción}

El presente artículo describe y explica los mecanismos y medidas que la Unión Europea ha puesto en marcha desde 2015 a raíz de la presentación de la Agenda Europea de Migración, redactada a consecuencia de la dramática situación vivida en las aguas del Mediterráneo. En propias palabras de la Comisión Europea (CE):

"La Comisión Europea ha presentado hoy una Agenda Europea de Migración en la que esboza tanto las medidas inmediatas que se adoptarán a fin de responder a la situación de crisis que existe en el Mediterráneo como los diversos pasos que deben darse en los próximos años para gestionar mejor la migración en todos sus aspectos" ${ }^{\prime \prime}$.

A partir de 2015 y hasta la firma del acuerdo con Turquía en marzo de 2016, Grecia fue el país que mayor número de entradas experimentó, 132.240 durante la primera mitad de 2015 según los datos aportados por Frontex². La CE desarrolló las medidas inmediatas y los cuatro pilares sobre los que se basarán las medidas especiales redactadas en la Agenda Europea de Migración a consecuencia de la llegada masiva de personas en busca de refugio a las costas griegas e italianas. Es por esto por lo que el presente artículo se centrará en el caso griego, debido a su actualidad y especialidad dada su situación geográfica, política y económica.

\section{Situación geográfica}

La situación geográfica griega es bastante peculiar si tenemos en cuenta diversos factores. Por un lado, la cercanía de varias islas griegas a la costa turca hace que las personas en busca de asilo elijan esta ruta desde que se aumentaran las medidas de seguridad en la frontera grecoturca a partir de 2010. Ese año Frontex desplegó su primer equipo Rapid Border Intervention Teams (RABIT ${ }^{3}$ ) a petición del ministerio griego de protección ciudadana, y más tarde en 2012 se terminó de erigir la valla de $12.5 \mathrm{~km}$ que separa el territorio griego del turco a lo ancho del río Evros en el norte de Grecia. Por otro lado está el hecho de que los países que limitan al norte con Grecia, como Albania y Macedonia, no son miembros de la Unión Europea (UE), así como el resto de los países de la antigua Yugoslavia excepto Croacia y Bulgaria que limitan con Grecia y que sí son miembros de la Unión pero no del espacio Schengen.

Grecia e Italia son las principales puertas de entrada a Europa, pero en el caso griego su situación geográfica complica la salida y llegada de las personas en busca de asilo a otros países de la UE, puesto que la ruta a seguir desde Grecia recorre distintos países de la península balcánica que, o bien no son miembros de la UE o no pertenecen al espacio Schengen. Situación que se traduce en mayor dificultad para atravesar los pasos fronterizos, especialmente desde la primera mitad del 2016 en la que varios países de la ruta de los Balcanes reforzaron y cerraron sus fronteras ${ }^{4}$.

\footnotetext{
1 Agenda Europea de Migración, comunicado de prensa: http://europa.eu/rapid/press-release_IP-15-4956_ es.htm

2 Datos extraídos del artículo de PARK, Jeanne, "Europe's migration crisis", New York: Council of Foreign Relations, 2015, ps. 311-325

3 Datos detallados sobre la primera misión del Frontex en Grecia aparecen explicados en el informe de la operación RABIT del 2010: http://frontex.europa.eu/assets/Attachments_News/fer_rabit_2010_screen_v6.pdf [Consultado el 27 de agosto de 2017]

${ }^{4}$ Hecho que explica Dimitris Dakaloglou en su artículo "Europe's last frontier: The spatialities of the refugee
} 


\section{Situación política y económica griega}

La gestión de los flujos migratorios por parte de Grecia viene marcada principalmente por directrices y decisiones de la CE que el Estado griego transpondrá en su legislación. La gestión e implementación de los programas de ayuda deberá respetar la legislación griega y armonizarse con ésta, ya que la mayoría de los fondos provienen de presupuestos especiales que la CE ha diseñado y destinado a la gestión de la situación migratoria en Grecia junto con la colaboración de agencias de la ONU como ACNUR, UNFPA, UNICEF y IOM entre otras, así como diferentes ONG internacionales ${ }^{5}$.

La crisis económica en la que está sumida Grecia desde 2009 la incapacita a la hora de destinar presupuestos del estado a la gestión de la situación migratoria. La "Troika" no deja mucho margen de decisión al gobierno griego, los tres memorándums de entendimiento y en especial el último 6 marcan las decisiones, compromisos y organización del gasto público a los que el gobierno griego debe obedecer para el desembolse de los fondos.

De este modo, cualquier medida y/o programa que se implemente en territorio griego y destinado a la atención de las personas solicitantes de asilo y refugiadas, no serán medidas adoptadas por el gobierno griego, serán programas y medidas diseñadas por instituciones internacionales y organismos supranacionales, que se llevarán a cabo en territorio griego en consonancia y respetando la legislación y gestión por parte de las autoridades griegas junto con los actores internacionales y locales en terreno.

La distribución de las personas refugiadas y solicitantes de asilo asentadas en los diferentes campos de refugiados repartidos por todo el territorio heleno, el acceso a diversos servicios y programas y las medidas excepcionales implementadas después de la firma del acuerdo UE-Turquía; muestran una clara imagen de la discriminación a la que se enfrentan las personas solicitantes de asilo dependiendo de su nacionalidad.

El acceso a diferentes programas y servicios e incluso a la solicitud de asilo, depende en muchas ocasiones en el caso griego actual, del país de origen al que pertenece la persona o donde tuviera su residencia habitual antes de llegar a Grecia. Existen diferentes casuísticas y situaciones. En el presente artículo se describirán medidas cuya implementación establecen realidades a las que se enfrentan diariamente dos de las nacionalidades más numerosas que han llegado a Grecia en los dos últimos años: la afgana y la pakistaní?.

crisis": http://www.tandfonline.com/doi/full/10.1080/13604813.2016.1170467. [Consultado el 27 de agosto de 2017]

5 Así pues la mayoría de los fondos y programas implementados en Grecia provienen de agencias de la ONU con presencia en territorio griego como OIM, UNICEF, ACNUR, UNFPA, ONG internacionales y agencias de la Unión Europea como EASO y FRONTEX. A su vez estos actores internacionales colaboran con actores locales como son las ONG, autoridades y servicios nacionales y/o locales. De este modo EASO apoya al servicio de asilo griego, ONG internacionales y agencias de la ONU cuentan con presencia en los centros de recepción y áreas urbanas para colaborar en las labores de gestión, protección e implementación de programas dirigidos a la población refugiada.

6 Página de la Comisión Europea donde se pueden consultar los memorándums: https://ec.europa.eu/info/files/ memorandum-understanding-greece-august-2015_en [Consultado el 20 de agosto de 2017]

7 Nota: En este artículo no se hace uso del término crisis de los refugiados porque no comparto ni estoy de acuerdo con el significado del mismo. Como apunta Xavier Alcaide, la palabra crisis tiene connotaciones negativas y es tremendista, además de responsabilizar a las personas que buscando refugio intentan llegar a Europa; en vez de poner el acento en las políticas migratorias, de asilo y acogida de los países de la Unión Europea. Dichas 


\section{Medidas especiales tomadas por la UE}

Desde el comienzo de la actual situación migratoria ${ }^{8}$ en Europa, se han tomado diversas medidas y puesto en marcha diferentes mecanismos excepcionales que pueden utilizarse para llevar a cabo acciones discriminatorias. Procedimientos como el Syrian Fast-Track, el programa de reubicación, los programas de alojamiento (cuyo acceso se basa en la elegibilidad de la persona para el programa de reubicación) y la firma acuerdos internacionales recientes, son varios de los ejemplos que se describen en el presente artículo.

\subsection{Fast-track sirio}

Una de las primeras medidas relacionada con la llegada masiva de solicitantes de asilo a territorio griego, puesta en marcha en 2014 por la CE para facilitar y agilizar la tramitación de las solicitudes de asilo de los nacionales sirios que se encuentren en posesión de su pasaporte, recibe el nombre de Syrian Fast-Track ${ }^{9}$. Este procedimiento acelerado se aplica en favor de las y los nacionales sirios y no acepta pasaportes de otras nacionalidades a no ser que la persona solicitante de asilo sea de origen palestino y tuviera su residencia habitual en Siria antes de su llegada a Grecia ${ }^{10}$.

Dicha medida debería extenderse a cualquier nacionalidad, ya que la posesión del pasaporte en sí mismo sirve de gran ayuda al funcionariado que procesa las solicitudes de asilo, como documento oficial que asegura la nacionalidad del o la solicitante de asilo y permite agilizar el procedimiento. El procedimiento se puso en marcha en 2014 basándose en las tasas de reconocimiento que ostentaba la nacionalidad siria, pues en Grecia en aquel momento la tasa de reconocimiento rondaba el $99 \%$.

La definición del término de refugiado que se hace en la Convención Sobre el Estatuto de los Refugiados de 1951 no hace referencia únicamente a refugiados de guerra ni se basa en tasas de reconocimiento, sino que habla claramente sobre temores fundados de persecución. De este modo, la interpretación que hace la Comisión Europea de dicha definición a la hora de implementar medidas extraordinarias olvida la idea básica de la misma, descrita en el Artículo 1 de la convención y que establece que toda persona en base a fundados temores de ser perseguida por motivos de raza, religión, nacionalidad, pertenencia a determinado grupo social u opiniones políticas, puede reconocerse como refugiada. Asimismo el Derecho

políticas son las que están en crisis. Por este motivo prefiero utilizar situación migratoria en Grecia o en territorio griego para hacer referencia a todo lo relacionado con la situación actual que viven las personas que llegan o se encuentran atrapadas en Grecia en busca de protección internacional.

8 Migraciones forzosas, debido a las condiciones en las que vivieran las personas que han abandonado sus países de origen o donde tuvieran su residencia habitual y por ello solicitan asilo en Grecia.

9 Desde el 23 de septiembre de 2014 se aplica el trámite acelerado a los ciudadanos sirios y apátridas que tenían su residencia habitual en Siria. El procedimiento acelerado sólo está disponible para los ciudadanos sirios y apátridas que tengan su residencia habitual en Siria y entren en territorio griego antes de la entrada en vigor de la Declaración UE-Turquía. Las solicitudes de las personas que lleguen después del 20 de marzo de 2016 se examinarán sobre la admisibilidad en los conceptos de "primer país de asilo" y "tercer país seguro", a menos que sus solicitudes se consideren admisibles y, por consiguiente, se refieran al procedimiento ordinario".

(Traducción de la autora). Fuente: AIDA, Asylum Information Database :http://www.asylumineurope.org/ reports/country/Greece/asylum-procedure/treatment-specific-nationalities [Consultado el 14 de abril de 2017]

${ }^{10}$ Instrucciones para solicitar asilo, sistema griego de asilo, ¿Qué es el Fast-track sirio y quiénes pueden solicitarlo?: http://asylo.gov.gr/en/wp-content/uploads/2016/10/Qandanswers_ENG_OCT_V6.pdf [Consultado el 14 de abril de 2017] 
de asilo ${ }^{11}$ es un derecho internacional de los Derechos Humanos que se contempla en la Carta de los Derechos Fundamentales de la Unión Europea y lo garantiza, diciendo así:

"Se garantiza el derecho de asilo dentro del respeto de las normas de la Convención de Ginebra de 28 de julio de 1951 y del Protocolo de 31 de enero de 1957 sobre el Estatuto de los Refugiados y de conformidad con la Constitución"12

Como explica Timothy J. Hatton ${ }^{13}$, para los países de destino, la ley que regula el asilo se basa en la Convención de 1951 sobre el Estatuto de los Refugiados. De este modo, se contemplan tres disposiciones clave haciendo hincapié la primera en la individualidad de cada solicitud.

La primera disposición clase se basa en la definición del término refugiado (artículo 1 de la Convención como se ha mencionado antes), la cual establece que la determinación del estatuto de refugiado debe hacerse caso por caso a la luz de las pruebas disponibles y de manera independiente. En segundo lugar, la cláusula de no devolución (artículo 33.1), exige que el solicitante de asilo no sea devuelto a las fronteras de un territorio en el que su vida o su libertad estén amenazadas. En la práctica, esto significa que algunos solicitantes no pueden ser devueltos, incluso si no se les ha concedido el estatuto de refugiado, hecho que ha proporcionado una base para formas subsidiarias de protección. El tercero es que la entrada o la presencia no autorizadas en el país no impide la admisión en el procedimiento de determinación del estatuto de refugiado ni perjudica el resultado de ese proceso (artículo 31 ) $^{14}$.

De este modo, vemos como el Fast-Track sirio es una iniciativa que agiliza únicamente los trámites del proceso de solicitud de asilo para las personas sirias. A simple vista se trata de una medida positiva, pero el hecho de que excluya al resto de nacionalidades y personas que provienen de países cuya situación se asemeja a la del Estado sirio, la dota de un cariz discriminatorio. Si el mismo procedimiento quedara abierto para toda persona que dispusiera de pasaporte y proviniese de países que la comunidad internacional ha reconocido como zona de guerra, entonces estaríamos hablando de una medida justa y no discriminatoria. Según el Instituto Internacional de Estudios Estratégicos, existen en la actualidad 40 conflictos armados en activo ${ }^{15}$.

\subsection{El programa de reubicación}

El programa de reubicación descrito en la Agenda Europea de Migración de $2015^{16}$ se basa

${ }^{11}$ Art.14 de la Declaración Universal de los Derechos Humanos: "1) En caso de persecución, toda persona tiene derecho a buscar asilo, y a disfrutar de él, en cualquier país".

12 Artículo de 18 de la Carta de los Derechos Fundamentales de la Unión Europea de 2007.

13 HATTON, Timothy J., "Refugees and Asylum seekers, the crisis in Europe and the future of policy", Economic Policy, 2017, vol. 32, no 91, ps. 447-496.

https://academic.oup.com/economicpolicy/article/32/91/447/4060670/Refugees-and-asylum-seekers-thecrisis-in-Europe [Consultado el 20 de agosto de 2017]

${ }^{14}$ Párrafo resumido y traducido por la autora, extraído de HATTON, Timothy J., "Refugees and asylum seekers... op.cit.

${ }^{15}$ Para informaciones detalladas sobre cada conflicto consultar en la base de datos del IISS: https://acd.iiss.org/ [Consultado el 23 de agosto de 2017]

${ }^{16}$ Explicación de los puntos más importantes de la Agenda Europea de la Migración relacionados con el programa de reubicación: "Responder a las llegadas masivas en el seno de la UE: reubicación. La propuesta incluirá un 
en el traslado de personas en necesidad de protección internacional de un estado a otro de la Unión Europea previsto en el artículo 78, apartado 3 del TFUE que puede activarse cuando uno o varios estados se enfrenten a una situación de emergencia originada por la afluencia de repentina de nacionales de terceros países.

Las nacionalidades que disfrutaban del acceso a dicho programa cambiaron el pasado 1 de abril, ya que solo pueden ser reubicados los nacionales de terceros países cuya tasa de reconocimiento de protección internacional (teniendo en cuenta todos los países integrantes de la Unión Europea) supere el 75\%. De este modo, las personas nacionales de Antigua y Barbuda, Bahréin, los países y territorios británicos de ultramar, Eritrea, Granada, Guatemala, Siria, Yemen y apátridas que anteriormente residían en uno de estos países tienen acceso y pueden solicitar formar parte del programa de reubicación en la actualidad ${ }^{17}$.

Nacionales afganos, pakistaníes, iraníes e iraquíes, principales nacionalidades que se encuentran en mayor número, además de la siria, que han llegado a Grecia en busca de asilo desde 2015, no tienen acceso a medidas y programas de los que debería disfrutar cualquier solicitante de asilo dentro de las fronteras de la Unión Europea. Estos mecanismos destinados a favorecer y agilizar solicitudes de nacionales de ciertos países conllevan acciones discriminatorias como el hecho de permitir abandonar territorio griego e italiano de forma regular a personas de ciertas nacionalidades y tener acceso a protección internacional en otro país de la UE.

Estos programas, al estar basados en las tasas de reconocimiento de protección internacional (porcentaje de casos a los que se les concede el estatus de refugiado o protección subsidiaria), hace que los casos sufran una suerte de generalización dependiendo de la nacionalidad del solicitante, cuando cada caso según el derecho internacional de los refugiados, debe cuidarse de ser estudiado con independencia como apuntan Hatton y Thomas Rutting ${ }^{18}$, quien advierte que el uso de las tasas de reconocimiento puede llegar a determinar las resoluciones de las solicitudes además de socavar la individualidad del derecho al asilo.

régimen de reparto temporal de las personas que tengan manifiestamente necesidad de protección internacional a fin de garantizar una participación equitativa y equilibrada de todos los Estados miembros en este esfuerzo común. El Estado miembro de acogida será responsable del examen de la solicitud de conformidad con las normas y garantías en vigor.

[...]A la espera de la puesta en práctica de estas dos medidas, los Estados miembros tendrán que dar muestras de solidaridad e intensificar sus esfuerzos para ayudar a los países situados en primera línea". http://eurlex.europa.eu/legal-content/ES/TXT/HTML/?uri=CELEX:52015DC0240\&from=EN [Consultado el 14 de abril de 2017]

17 Fuente: https://www.easo.europa.eu/questions-and-answers-relocation [Consultado el 14 de abril de 2017]

${ }^{18}$ RUTTIG, Thomas, "Preamble: Afghans as a second-class refugees in parts of Europe", En CIDOB Monografías, Telling the story, sources of tension in Afghanistan \& Pakistan: A regional perspective (2011-2016), octubre 2016, ps. 103-104. 
Tabla. Tasas de reconocimiento de protección internacional por nacionalidades ${ }^{19}$.

\begin{tabular}{|c|c|c|c|}
\hline \multicolumn{4}{|c|}{ 1st Instance Procedures - Recognition Rates $(\%)^{2}$ (Countries with more than 60 Decisions in substance) } \\
\hline \multicolumn{2}{|c|}{10 Countries of Origin with the highest recognition rates } & \multicolumn{2}{|c|}{10 Countries of Origin with the lowest recognition rates } \\
\hline SYRIA & $99.6 \%$ & DOMINICAN REPUBLIC & $2.6 \%$ \\
\hline YEMEN & $93.7 \%$ & PAKISTAN & $2.5 \%$ \\
\hline PALESTINE & $92.2 \%$ & ALGERIA & $1.5 \%$ \\
\hline STATELESS & $83.9 \%$ & SENEGAL & $1.2 \%$ \\
\hline ERITREA & $80.7 \%$ & ARMENIA & $0.9 \%$ \\
\hline SOMALIA & $74.0 \%$ & GHANA & $0.7 \%$ \\
\hline IRAQ & $66.6 \%$ & ALBANIA & $0.2 \%$ \\
\hline SUDAN & $58.8 \%$ & GEORGIA & $0.0 \%$ \\
\hline AFGHANISTAN & $58.5 \%$ & RUSSIA & $0.0 \%$ \\
\hline IRAN & $51.7 \%$ & MOLDOVA & $0.0 \%$ \\
\hline
\end{tabular}

\section{Acceso al asilo y diferencia de trato dependiendo del país de origen}

Los pasos a seguir y el itinerario del proceso dependen de la nacionalidad del solicitante, pues en base a ello recibirá un tratamiento diferenciado para que su solicitud sea procesada de forma más rápida o más lenta, siendo los tiempos de espera más dilatados para determinadas nacionalidades.

Desde el 20 de marzo de 2016 y a causa de la firma de la Declaración UE-Turquía, se estableció un procedimiento acelerado fronterizo en lo que respecta a la tramitación de las solicitudes de asilo y las llegadas de las personas en busca de refugio a las islas griegas.

Como se menciona en el procedimiento fronterizo acelerado, la implementación del artículo 60.4 L 4375/2016 del acuerdo con Turquía ha variado en función de la nacionalidad de los solicitantes interesados.

Inicialmente se concedió prioridad al registro y tramitación de los casos sirios. Desde el verano de 2016, los solicitantes procedentes de países considerados con bajos niveles de reconocimiento, como Marruecos, Argelia, Túnez, Pakistán o Bangladesh, comenzaron a someterse a evaluaciones por mérito ${ }^{20}$ y fueron entrevistados por el Servicio de Asilo y $\mathrm{EASO}^{21}$.

El registro y el procesamiento de las nacionalidades que ostentan una tasa de reconocimiento superior al $25 \%$, como la afgana e iraquí, se inició a partir de diciembre de 2016.

Una vez la persona haya comenzado el proceso de tramitación de su solicitud de

${ }^{19}$ Fuente: Servicio griego de asilo: http://asylo.gov.gr/en/wp-content/uploads/2017/04/Greek_Asylum_Service_ Statistical_Data_EN.pdf [Consultado el 16 de abril de 2017]

${ }^{20}$ ACNUR. Nota sobre la Carga y el Mérito de la Prueba en las Solicitudes de Asilo. (...) "7. En el contexto de la responsabilidad del solicitante de probar los hechos que sustentan su solicitud, el término "mérito de la prueba" significa el umbral que debe alcanzar el/la solicitante para persuadir al examinador sobre la verdad de sus afirmaciones respecto a los hechos acontecidos. Los hechos que deben probarse son aquellos relacionados con los antecedentes y experiencias personales del solicitante, que presumiblemente han originado el temor de persecución y la consecuente falta de disposición a acogerse a la protección del país de origen". http://www. acnur.org/t3/fileadmin/Documentos/BDL/2002/1906.pdf [Consultado el 16 de abril de 2017]

${ }^{21}$ Oficina Europea de Apoyo al Asilo: Esta agencia funciona como centro de referencia en materia de asilo; contribuye al desarrollo del sistema europeo común de asilo al facilitar, coordinar y reforzar la cooperación práctica entre los Estados miembros en los múltiples aspectos del asilo, y ayuda a los Estados miembros a cumplir sus obligaciones europeas e internacionales de ofrecer protección a las personas que lo necesiten. 
asilo o petición de reubicación, ésta será trasladada a la península quedando a la espera de la resolución de su solicitud, la cual depende del Servicio de Asilo Griego junto con el apoyo de EASO. De acuerdo con la ley griega 4375 de 2016 el examen de una solicitud de asilo en primera instancia en territorio griego, no debería exceder los seis meses para el procedimiento ordinario y los tres para el procedimiento acelerado (fast-track). Tiempos que no suelen cumplirse teniendo en cuenta la cantidad de solicitudes y el personal de la oficina de asilo griega22.

Tal y como se ha descrito anteriormente, el régimen de reubicación sólo está disponible para los solicitantes que "necesiten manifiestamente una protección internacional", que hubieran ingresado en el territorio griego entre el 16 de septiembre de 2015 y el 19 de marzo de 2016 y que la nacionalidad del o la solicitante experimente una tasa de reconocimiento medio del $75 \%$ o más para toda la Unión Europea. Así, sólo las nacionalidades específicas determinadas ${ }^{23}$ por los datos recogidos y actualizados trimestralmente por Eurostat, pueden ser trasladadas a otro estado miembro de la UE.

\section{Acceso a programas de vivienda y alojamiento}

Desde la apertura de los centros de recepción ${ }^{24}$ la mayoría de sus ocupantes en la región de Ática, la Grecia continental y las islas cercanas a la costa turca, proceden de Afganistán, Pakistán e Irak.

Las medidas de seguridad en las islas se reforzaron a partir del 20 de marzo de 2016 a consecuencia de la firma del acuerdo con Turquía dificultando la salida hacia Grecia continental de nacionales afganos, iraníes y pakistaníes, debido al difícil acceso a solicitar asilo y la consecuente prohibición por parte de las autoridades de abandonar las islas sin el documento de registro completo que admite a trámite su solicitud a expensas de la decisión final (tarjeta identificativa del solicitante de asilo).

La situación actual obliga a la mayoría de personas de origen afgano y pakistaní a permanecer en las islas como muestran los datos que aparecen en los perfiles técnicos oficiales de los centros de recepción temporal redactados por ACNUR. Esto se debe al difícil acceso a solicitar asilo o a la dilatación de los tiempos que caracterizan los trámites relacionados con las solicitudes de asilo en Grecia. Es cierto que el proceso es largo para cualquier solicitante de asilo, pero mecanismos como el Syrian Fast-track y programas como el de reubicación (a los que no tienen acceso todas las nacionalidades), ayudan a agilizar el proceso.

\subsection{Alojamiento en zonas urbanas}

El pasado diciembre ACNUR alcanzó su objetivo de alojar a 20.000 personas a través de su plan de alojamiento financiado por la Comisión Europea. El programa de ACNUR proporciona

22 Una de las razones por la que el servicio de asilo griego necesita del apoyo de EASO.

${ }^{23}$ No figuran la afgana, pakistaní y la somalí entre otras. Países que como explicaremos más adelante se encuentran en situaciones críticas caracterizadas por altos grados de violencia.

${ }^{24}$ Algunos, especialmente los desplegados en las islas, son campos de retención puesto que las personas que residen en ellos no tienen derecho a abandonar las islas a raíz del acuerdo que la Unión Europea estableció con Turquía. Así lo denuncia el Informe de Amnistía Internacional sobre la situación en las islas griegas y ruta balcánica después del acuerdo UE-Turquía y el cierre de fronteras: https://www.amnesty.org/en/latest/ news/2017/03/the-eu-turkey-deal-europes-year-of-shame/ [Consultado el 16 de abril de 2017] 
alojamiento únicamente a los candidatos al programa de reubicación y a los solicitantes de asilo considerados especialmente vulnerables. Por lo tanto, las nacionalidades que no tienen acceso al programa de reubicación tampoco lo tienen al programa de alojamiento gestionado por ACNUR a no ser que se traten de casos especialmente vulnerables ${ }^{25}$. Entre las nacionalidades que no tenían acceso a dicho programa se encontraban una vez más las personas de origen afgano, pakistaní, iraní o palestino, entre otras.

Alrededor del $58 \%$ de los lugares de alojamiento son pequeños apartamentos temporales, administrados por contrapartes de ACNUR. Otro $25 \%$ corresponde a habitaciones de hotel a precios especiales. Este programa financiado principalmente por la Comisión Europea cuenta además con edificios alquilados destinados a la acogida de solicitantes de asilo, así como la posibilidad de alojarse con familias anfitrionas griegas. Los beneficiarios también reciben servicios psicosociales, legales y de interpretación.

\subsection{2. ¿Quién vive en los centros de recepción? ¿Qué nacionalidades predominan?}

En Grecia continental sólo en la región de Ática existen doce centros de recepción cuya población se distribuye de la siguiente manera.

\begin{tabular}{|c|c|c|c|c|c|}
\hline $\begin{array}{l}\text { Centro de } \\
\text { recepción }\end{array}$ & $\begin{array}{r}\text { Población } \\
\text { Afgana }\end{array}$ & $\begin{array}{r}\text { Población } \\
\text { Siria }\end{array}$ & $\begin{array}{r}\text { Población } \\
\text { Iraquí }\end{array}$ & $\begin{array}{l}\text { Población } \\
\text { Pakistaní }\end{array}$ & Otras \\
\hline Elefsina & $99 \%$ & $1 \%$ & & & \\
\hline Eleonas I y II & $55 \%$ & $35 \%$ & & & $10 \%$ \\
\hline Eleonas III & $20 \%$ & $75 \%$ & $5 \%$ & & \\
\hline Elliniko I & $98 \%$ & & & $1 \%$ & $1 \%$ \\
\hline Elliniko II & $99 \%$ & & & & $1 \%$ \\
\hline Elliniko III & $98 \%$ & & & $1 \%$ & $1 \%$ \\
\hline Lavrio & $3 \%$ & $96 \%$ & & $1 \%$ & \\
\hline Rafina & $48 \%$ & $52 \%$ & & & \\
\hline Schisto & $95 \%$ & & & & $5 \%$ \\
\hline Malakasa & $98 \%$ & & & & $2 \%$ \\
\hline Skaramagkas & $10 \%$ & $65 \%$ & $25 \%$ & & \\
\hline
\end{tabular}

Datos extraídos del informe sobre el perfil de los centros de recepción en toda Grecia que ACNUR

actualiza asiduamente ${ }^{26}$.

De los doce centros de recepción en la región de Ática sólo Eleonas cumple con las

${ }^{25}$ Existe una lista de criterios de vulnerabilidad prevista en la legislación nacional (griega) pertinente y de acuerdo con el artículo 14(8) L 4375/2016, establece que "Como grupos vulnerables se considerarán ... a) los menores no acompañados, b) las personas con discapacidad o que padecen una enfermedad incurable o grave, c) las personas mayores, F) víctimas de torturas, violaciones u otras formas graves de violencia o explotación psicológica, física o sexual, personas con trastornos postraumáticos, en particular supervivientes y familiares de menores de edad, víctimas de naufragios, g) víctimas de la trata de seres humanos".

Los solicitantes recién llegados que se consideren vulnerables de acuerdo con la definición del artículo 14(8) L 4375 no tienen acceso al Procedimiento Fronterizo Rápido (Fast-track) y sus reclamaciones son consideradas admisibles desde la firma del Acuerdo UE-Turquía.

${ }^{26}$ Datos extraídos del informe de ACNUR, Site Profiles-Greece document. Documento que proporciona información detallada sobre los centros de recepción en todo el territorio griego. La información se actualiza mensualmente y es recogida de diversas formas, con la colaboración del personal de ACNUR que trabaja en los centros, los coordinadores de los centros y la policía griega entre otros actores. 
condiciones mínimas de habitabilidad donde sea posible cubrir las necesidades básicas de las personas que residen en el centro de recepción, según la afirmación realizada por la Comisión Nacional de los Derechos Humanos después de visitar los centros de recepción ${ }^{27}$.

Varias organizaciones internacionales denunciaron las condiciones deplorables en las que vivían las personas que residían en diversos centros de la península y más concretamente en región de Ática, como Malakasa y Elliniko, donde la mayoría de los residentes son de origen afgano.

La misión de Médicos sin Fronteras en Grecia explicó su preocupación por la situación en la que se encontraban las personas que residen en dichos centros. En Grecia continental se tardó y, en algunos casos, no llegó la preparación de los espacios para hacer frente a las condiciones climáticas más extremas que se dieron durante el invierno (Winterization), especialmente en zonas como el norte de Grecia y Malakasa, donde cada año se registran temperaturas que rondan los cero grados. Durante el invierno se dieron fuertes nevadas en el norte de Grecia que enterraron campos bajo la nieve, quedándose sin agua ni calefacción durante días y experimentando problemas graves en relación al suministro de energía ${ }^{28}$.

Con respecto a Elliniko (como hemos visto en la tabla, la mayoría de las personas alojadas eran de origen afgano), el pasado febrero las personas solicitantes de asilo que residen en dicho centro protagonizaron una huelga de hambre para denunciar las condiciones en las que viven y lo inadecuado de las instalaciones. Reclamaban agua caliente, calefacción, alimentos, pañales, ropa y leche para bebés, además de la falta de intérpretes que puedan acompañarles al hospital, así como que se cubran los costes de su traslado a los mismos ${ }^{29}$.

\section{2. ¿Qué ocurre en las islas?}

La situación en los centros de recepción es más complicada debido a la tensión que se vive desde la firma del acuerdo UE-Turquía y las medidas implementadas. En las islas no sólo encontramos centros de recepción e identificación, sino centros de retención. Varios de los Hotspots $^{30}$ (centros de identificación) actúan como centros de retención ya que las personas recién llegadas se encuentran retenidas de facto dentro de las instalaciones del centro durante un período de 25 días. Una vez pasado ese periodo, las personas recién llegadas pueden entrar y salir de las instalaciones del centro libremente. Estos centros hacen las veces de centros

27 National Comission for Human Rights, "Living Conditions in Reception Centres for Migrants and Refugees", diciembre de 2016: http://www.nchr.gr/images/pdf/apofaseis/prosfuges_metanastes/kentra_filoxenias_ autopsia.pdf [Consultado el 18 de abril de 2017]

${ }^{28}$ https://www.msf.gr/magazine/einai-ntropi-i-eikona-poy-vlepoyme-simera-stoys-kataylismoys-gia-tis-ellinikeskai [Consultado el 18 de abril de 2017]

29 "CNN Grecia informó que Mouzalas (Ministro de Migración griego) se reunió con representantes de los migrantes y refugiados de Elliniko. Sus demandas principales incluyeron agua caliente de manera asidua, lavadoras, comida, atención médica de mejor calidad y acceso a la educación formal para sus hijos". (Traducción de la autora). https://newsthatmoves.org/en/hunger-strike-and-tensions-at-elliniko/ [Consultado el 19 de abril de 2017]

${ }^{30}$ El Hotspot approach fue puesto en marcha por la UE con el fin de establecer mecanismos de solidaridad que puedan hacer frente a situaciones de emergencia, como las llegadas masivas de nacionales de terceros países. Lo explica el Real Instituto Elcano en uno de sus informes "Los hotspot, un eslabón débil en la gestión de la crisis de los refugiados" cuando explica que "El objetivo del hotspot approach es facilitar la reubicación temporal de los solicitantes de asilo a través del apoyo operativo en la identificación, registro, toma de huellas dactilares y entrevistas a dichos solicitantes. También deben apoyar al Estado en la gestión del retorno de los que no tienen derecho a solicitar asilo". 
de retención y recepción, ya que pasados los días en los que las personas se encuentran retenidas el mismo centro pasa a ser su alojamiento.

Además de las difíciles condiciones en las que han vivido durante el invierno las personas que se encuentran alojadas en estos centros, los hotspots y centros de recepción en las islas podrían describirse como un limbo sobre la tierra, en donde se acumulan las tensiones fruto de la espera, la retención y la incertidumbre que sufren las personas retenidas en las islas griegas.

Durante 2016 la Agencia de la Unión Europea por los Derechos Fundamentales registró una serie de incidentes graves que ocurrieron en los hotspots griegos:

"De acuerdo con el apartado 4 del artículo 18 de la Directiva sobre las condiciones de acogida, los Estados miembros deben adoptar las medidas adecuadas para prevenir las agresiones y la violencia de género incluyendo agresiones sexuales y el acoso. En los hotspots se han denunciado incidentes graves, incluyendo violaciones y otros crímenes violentos, así como intentos de suicidio y situaciones de disturbios graves. El respeto y cuidado de la seguridad en los hotsopts ha sido particularmente difícil en Grecia, debido al hacinamiento, la prolongada estancia y la práctica de devoluciones a Turquía directamente desde los hotspots. La siguiente tabla describe varios incidentes significativos en los hotspots griegos entre abril y noviembre de $2016^{\prime \prime}$.

${ }^{31}$ Tabla y párrafo traducidos por la autora y extraído del Informe de la Agencia de la Unión Europea de Derechos Fundamentales: "Opinion of the European Union Agency for Fundamental Rights on fundamental rights in the 'hotspots' set up in Greece and Italy". 


\begin{tabular}{|c|c|c|}
\hline Fecha & Incidente & Hotspot \\
\hline 24.11 .2016 & $\begin{array}{l}\text { Muerte de dos personas que residían en el hostpot en una } \\
\text { explosión accidental. Varias personas fueron hospitalizadas. }\end{array}$ & Lesbos \\
\hline 09.11 .2016 & $\begin{array}{l}\text { Las tiendas y pertenencias de las personas que residían } \\
\text { en el hostspot sufrieron graves daños a consecuencia de } \\
\text { lluvias torrenciales. Los residentes inician una protesta } \\
\text { para reclamar ropa seca y tiendas nuevas. }\end{array}$ & Samos \\
\hline 26.10 .2016 & $\begin{array}{l}\text { Las solicitantes de asilo protestan por los retrasos en el } \\
\text { examen de sus solicitudes de asilo. Según la denuncia, una } \\
\text { mujer ataca al personal de EASO. }\end{array}$ & Chios \\
\hline 24.10 .2016 & $\begin{array}{l}\text { Disturbios. Solicitantes de asilo prenden fuego a las } \\
\text { instalaciones de EASO. }\end{array}$ & Lesbos \\
\hline 20.10 .2016 & $\begin{array}{l}\text { Los solicitantes de asilo bloquean la entrada del hotspot } \\
\text { protestando por los retrasos en el examen de sus solicitudes } \\
\text { de asilo y la estancia prolongada en la isla. }\end{array}$ & Quíos \\
\hline 08.10 .2016 & Violación de un marroquí de 25 años por tres argelinos. & Lesvos \\
\hline 26.09 .2016 & $\begin{array}{l}\text { Un joven afgano intenta suicidarse después de recibir la } \\
\text { resolución negativa a su solicitud. }\end{array}$ & Quíos \\
\hline 25.09 .2016 & $\begin{array}{l}\text { Violación de un menos de } 16 \text { años perpetrada por otros } \\
\text { cuatro chicos. }\end{array}$ & Lesbos \\
\hline 04.09 .2016 & Personas alojadas en el hotspot incendian el campo. & Lesbos \\
\hline 07.07 .2016 & $\begin{array}{l}\text { Violentos enfrentamientos entre menores en el hotspot. } \\
\text { Cinco menores no acompañados son trasladados al hospital } \\
\text { mientras que otros se dan a la fuga. }\end{array}$ & Leros \\
\hline 09.07 .2016 & Personas alojadas en hotspot atacan a oficiales de policía. & Leros \\
\hline 28.06 .2016 & $\begin{array}{l}\text { Disturbios, las personas alojadas en el hotspot protestan } \\
\text { por las condiciones de vida, atacando al director de la } \\
\text { policía y al alcalde. Conflictos con los lugareños }\end{array}$ & Leros \\
\hline 02.06 .2016 & Intento de suicidio de una mujer Yazidi. & Samos \\
\hline 01.06 .2016 & $\begin{array}{l}\text { Se desata un incendio a raíz de los enfrentamientos entre } \\
\text { personas de diferentes nacionalidades. Las familias tienen } \\
\text { que escapar del campamento y pasar la noche fuera. }\end{array}$ & Lesbos \\
\hline 26.05 .2016 & Intento de suicidio de un hombre afgano. & Quíos \\
\hline 26.04 .2016 & $\begin{array}{l}\text { Disturbios, tensiones que comienzan en la sección de } \\
\text { menores no acompañados. }\end{array}$ & Lesbos \\
\hline 15.04 .2016 & Violación de un menor de 13 años. & Quíos \\
\hline 06.04 .2016 & Un hombre pakistaní amenaza con suicidarse. & Lesbos \\
\hline
\end{tabular}

\section{La declaración UE-Turquía y los nuevos programas de retorno e integración en los países de origen}

La Organización Internacional para las Migraciones (OIM) ha puesto en marcha nuevos programas de retorno voluntario y reintegración de las y los nacionales de terceros países en sus países de origen. Esta vez la pakistaní, argelina y marroquí son las nacionalidades que más se han beneficiado del programa. Prueba de ello es la cantidad de retornos voluntarios que se han realizado a Pakistán ${ }^{32}, 1.117$, siendo la nacionalidad que más retornos voluntarios

\footnotetext{
${ }^{32}$ Comunicado de prensa de la OIM (Organización Internacional para las Migraciones, siglas en inglés): "Desde junio de 2016 un gran número de migrantes han regresado voluntariamente a su país de origen. Hecho muy importante, ya que esas personas volvieron a casa de forma segura, donde la OIM les da la bienvenida y
} 
ha protagonizado.

Varias organizaciones han denunciado la falta de acceso a solicitar asilo así como falta de acceso a la información, informaciones vitales y necesarias para personas que llegan a Grecia buscando solicitar asilo.

\subsection{Declaración UE-Turquía}

¿Qué se acordó en la Declaración UE-Turquía33?

En relación con la gestión de las llegadas de personas en busca de asilo a las islas griegas, se acordaron las siguientes acciones. Primero, la devolución de las islas griegas a Turquía de las personas solicitantes de asilo y migrantes cuyas solicitudes sean declaradas inadmisibles a partir del 20 de marzo de 2016. Segundo, el reasentamiento de las personas solicitantes de asilo de origen sirio que se encuentren en Turquía. Por cada persona siria que sea devuelta a Turquía desde las islas griegas, otra persona de origen sirio será reasentada directamente en la UE desde Turquía. Tercero, la adopción por parte de Turquía de todas las medidas necesarias para evitar nuevas rutas marítimas o terrestres de migración irregular.

Nuevamente, únicamente las personas de origen sirio disfrutarán de la opción de ser reasentadas desde Turquía, tal y como afirmó Florian Furtak ${ }^{34}$ al explicar las clausulas de la declaración UE-Turquía antes de entrar en vigor en 2016. Turquía se comprometerá a mejorar las condiciones de vida de los refugiados de mayoría siria en el país y adoptar medidas especiales para asegurar la disminución de los flujos de personas en busca de asilo en Europa en el futuro 35 .

Apoyándome en las afirmaciones de Timothy Hatton, desde 2014 la ruta TurquíaEgeo-Balcanes comenzó a ser la más utilizada (por personas de diversas nacionalidades), aprovechando la mayor flexibilidad de las fronteras y controles tanto en el mar Egeo como en la ruta de los Balcanes en comparación con la ruta libia. ${ }^{36}$ Además, aunque se haya puesto la atención en los conflictos de oriente próximo, no hay que pasar por alto los conflictos enquistados en el continente africano que llevan generando refugiados durante años. Un ejemplo a destacar, es el de las personas atrapadas en campos de refugiados como el de Dadaab al norte de Kenia, siendo uno de los campos de refugiados que más personas aloja del mundo, 330.000 (en su mayoría refugiados somalíes). Dadaab difícilmente provee las necesidades básicas y la seguridad de sus habitantes se ve seriamente amenazada sistemáticamente.

les apoya en el proceso de reintegración ", dijo Giannis Mouzalas, Ministro de Política Migratoria en el evento informativo celebrado en la Secretaría General de Comunicación Griega. Además, Pakistán encabeza la lista de países de origen con 1.117 retornos voluntarios, seguidos por Irak (918), Afganistán (871), Georgia (456) y Argelia con 338 retornos voluntarios. Los datos mencionados corresponden al período comprendido entre junio de 2016 y marzo de 2017". Traducción de la autora.

${ }^{33}$ Fuente: "Implementing the EU-Turkey Statement - Questions and Answers: European Commission-Fact sheet". Traducción de la autora.

${ }^{34}$ FURTAK, T. Florian, "The refugee crisis-A challenge for Europe and the world", Journal of civil \& legal sciences, vol 5, 2015, Issue 1.

${ }^{35}$ Ibídem

${ }^{36}$ HATTON, Timothy J., "Refugees and Asylum seekers, the crisis in Europe and the future of policy", Economic Policy, 2017, vol. 32, no 91, ps. 447-496. 
Como apunta Nassim Majidi ${ }^{37}$, las personas solicitantes de asilo de origen somalí representaban la nacionalidad más numerosa en Noruega y Dinamarca hasta el 2015. Ese año se presentaron dentro de las fronteras de la Unión Europea 21.050 solicitudes de asilo de personas de origen somalí. Entre las nacionalidades que llegan a Europa en busca de asilo además de las personas procedentes de países de oriente próximo y medio, encontramos la eritrea con 34.130 solicitudes en 2015, la pakistaní 48.015 y la bangladesí 18.865 entre otras para ése mismo año ${ }^{38}$. De este modo podemos observar que tanto la ruta Libia-Italia como la Turquía-Grecia no sólo es usada por las personas provenientes del conflicto sirio, sino de otros lugares donde se violan Derechos Humanos y generan personas refugiadas que huyen a Europa en busca de protección internacional.

\section{Deportaciones a Turquía}

De las 777 deportaciones que se han realizado a Turquía desde la firma del acuerdo UETurquía (de abril de 2016 a enero de 2017), 40439 eran nacionales pakistaníes, seguidos de nacionales argelinos y afganos. Desde la firma del acuerdo y el establecimiento del Hotspot en Kos (94\% de la población paquistaní) sus residentes esperan en su mayoría la deportación a Turquía o el retorno a Pakistán a través de los programas de retorno voluntario (gestionados por la OIM), debido a las condiciones del acuerdo UE-Turquía, el difícil acceso a la información y la baja tasa de reconocimiento que experimenta la población pakistaní.

\subsection{Acuerdos de retorno voluntario Afganistán y Pakistán}

Existen muchos países cuya situación complicada, violenta y frágil hace que sus civiles no sientan la protección del estado e incluso su propia vida corra peligro y por estos motivos se vean forzados a desplazarse. Partiendo del hecho de que ningún país goza de una importancia mayor que otro y de la imposibilidad de describir aquí todos los conflictos y zonas críticas que ocurren alrededor del globo, este apartado se centrará únicamente en los casos de Afganistán y Pakistán ya que son dos de las nacionalidades más numerosas que en los tres últimos años han arribado a Grecia en busca de protección internacional.

Los nacionales afganos son ahora el segundo grupo de solicitantes de protección internacional más numeroso en la UE (más de 175.000 en 2016) por lo que Afganistán es un país de origen muy importante y a tener en cuenta. A lo largo de 2016 los retornos voluntarios asistidos aumentaron considerablemente llegando a casi las 6.000 personas. Deberían aumentarse los esfuerzos de todos los estados miembros de la UE para reducir el elevado número de afganos que siguen estancados en los países de la ruta de los Balcanes Occidentales y reubicarlos en países de la Unión como ocurre con las nacionalidades con acceso al programa de reubicación.

La Comisión Europea colabora con los países fronterizos y con las autoridades afganas

37 MAJIDI, Nassim, "Afghan and Somali (post-) conflict migration to the EU", Forced Migration Review, no 51, 2016, p. 32.

38 Datos extraídos del informe: "The Refugee Crisis Through Statistics": European Stability Initiative. 2017: http://www.esiweb.org/pdf/ESI\%20-\%20The\%20refugee\%20crisis\%20through\%20statistics\%20-\%2030\%20 Jan\%202017.pdf. [Consultado el 25 de agosto de2017]

39 Según el informe de Deportation Global Information Project: http://postdeportation.org/2017/02/the-eu-turkeydeal-what-happens-to-people-who-return-to-turkey/ [Consultado el 21 de abril de 2017] 
para avanzar en este ámbito. De este modo, la UE firmó un acuerdo con Afganistán en octubre de 2016 llamado "Joint Way Forward on Migration Issues between Afghanistan and EU"40, además de acuerdos bilaterales con diferentes estados miembros. En dichos acuerdos la UE afirma que apoyará la reintegración sostenible de los ciudadanos afganos que han regresado de Pakistán e Irán a Afganistán, facilitando el acceso a los servicios de apoyo a los medios de vida y la cohesión social en las comunidades de acogida. Se tendrá en cuenta un enfoque regional para apoyar la protección y la reintegración sostenible de los nacionales afganos desplazados ${ }^{41}$. Mi pregunta en este sentido pone el foco en este tipo de acuerdos y más concretamente en cómo puede la UE firmar un acuerdo de devolución con Afganistán, un país en guerra reconocido por la ONU y varios estados pertenecientes a la UE.

El reconocimiento por parte de la UE de desplazados afganos en países cercanos y la colaboración con los mismos para que la reintegración de los nacionales afganos sea posible, supone una increíble paradoja en la que de manera indirecta la UE reconoce a la población afgana su condición de desplazados en otros países como Pakistán e Irán.

\section{Situación en Afganistán}

Naciones Unidas tiene su propia misión en Afganistán, conocida bajo las siglas de UNAMA. Las misiones de Naciones Unidas creadas específicamente para abordar la situación en un país concreto suelen establecerse en zonas de guerra, de profunda inestabilidad política y en zonas que viven momentos de transición. En los informes anuales y cuatrimestrales de UNAMA sobre la evolución de la situación en Afganistán, se describe al mismo como zona de conflicto armado ${ }^{42}$.

Las bajas tasas de reconocimiento de la población afgana y el trato que se les da por parte de los estados miembros se contrapone a la idea que tienen estos sobre Afganistán, puesto que ninguno de los países que conforman la UE aconseja a sus ciudadanos viajar al mismo.

\footnotetext{
40 Nota introductoria del acuerdo entre la Unión Europea y Afganistán: "La Unión Europea y la República Islámica del Afganistán se enfrentan desafíos sin precedentes relacionados con refugio y migración. Abordarlos requiere solidaridad, determinación y esfuerzos colectivos. Esta iniciativa conjunta refleja el compromiso conjunto de la UE y el Gobierno del Afganistán de intensificar su cooperación para abordar y prevenir la migración irregular y el retorno de los migrantes irregulares que tras el examen de todas las leyes y procedimientos jurídicos internacionales pertinentes no pueden obtener el estatuto de protección internacional como parte de la asociación entre el Gobierno del Afganistán y la UE mediante esfuerzos coordinados. Con este fin, el Gobierno del Afganistán y la UE acordaron iniciar un Diálogo de Alto Nivel sobre la Migración".

[...]Este acuerdo identifica una serie de medidas que deben adoptar urgentemente la UE y el Gobierno del Afganistán con el objetivo de establecer un proceso rápido y eficaz para el regreso digno y organizado a Afganistán de los ciudadanos afganos que no cumplen con las condiciones de entrada, presencia o residencia en el territorio de la UE, además de facilitar su reintegración en el Afganistán a través de la cooperación". Traducción de la autora. Fuente original:

https://eeas.europa.eu/sites/eeas/files/eu_afghanistan_joint_way_forward_on_migration_issues.pdf [Consultado el 20 de abril de 2017]

${ }^{41}$ Informaciones extraídas del documento "Third Progress Report on the Partnership Framework with third countries under the European Agenda on Migration":https://eeas.europa.eu/sites/eeas/files/com_2017_205_f1_report_ from_commission_en_v8_p1_880005_0.pdf [Consultado el 21 de abril de 2017]

42 La UNAMA emprende una serie de actividades encaminadas a reducir al mínimo las repercusiones del conflicto armado en la población civil, entre ellas: la vigilancia independiente e imparcial de los incidentes de pérdida de vidas o lesiones a civiles, protección de los civiles afectados por el conflicto armado e iniciativas para promover el cumplimiento del derecho internacional humanitario, los Derechos Humanos y la Constitución y las leyes de Afganistán entre todas las partes en el conflicto "

Traducción realizada por la autora de la descripción de la misión de UNAMA: https://unama.unmissions.org/ protection-of-civilians-reports [Consultado el 20 de abril de 2017]
} 
La embajada de España afirma al respecto de la situación de inseguridad en el país: "Afganistán es un país en guerra. La comunidad internacional está expuesta a los secuestros oportunistas y todos, ciudadanos nacionales y extranjeros, están expuestos a todo tipo de atentados terroristas. Tanto en las grandes ciudades, incluida Kabul, como en las provincias, el nivel de inseguridad es muy elevado, con riesgo cierto para la propia vida. [...]El deterioro de la seguridad se ha agravado fuertemente los últimos meses y está previsto que se agrave aún más durante los próximos meses ${ }^{1 / 43}$.

La embajada británica en el país también recomienda a sus conciudadanos: "Existe un alto riesgo de amenaza terrorista y los métodos específicos de ataque están evolucionando y aumentando en sofisticación. Existe alto riego de secuestro en todo el país. Los viajes por carretera en todo el país, pero particularmente fuera de la capital Kabul son extremadamente peligrosos. Busque seguridad profesional para todos sus viajes y considere el uso de vehículos blindados" 44 .

A su vez, la embajada de Italia sostiene:

"En los últimos meses, la situación de seguridad se ha deteriorado significativamente en todas las partes del país, incluyendo ciudades importantes (Kabul, Herat, Mazar-e-Sharif, Jalalabad, Kandahar y Kunduz). Las áreas sur y sureste siguen siendo críticas y la situación se ha agravado también en el norte y oeste del país"45.

Recientemente la experta legal Alice Févre, que trabaja para el Foro Griego de los Refugiados en Atenas (Greek Forum of Refugees), escribió un artículo sobre como describen la situación en Afganistán diferentes países de la UE y cómo es posible que la tasa de reconocimiento de nacionales afganos varíe tanto de un país a otro. A consecuencia de esto el conjunto del porcentaje total para toda la Unión no es muy elevado como apunta el artículo46:

"En 2015 Francia concedió protección internacional al 80,3\% de los solicitantes de asilo afganos en su territorio. El mismo año Alemania concedió protección al $77,6 \%$ de los afganos, así como Bélgica al 77,3\% y Grecia al 60,5\%".

La pregunta final que se hace Févre en su artículo resulta bastante esclarecedora, "En consecuencia, pedimos a cada gobierno de la UE que responda conscientemente: Si Afganistán no es seguro para los ciudadanos de la UE, ¿cómo podría ser seguro para los

${ }^{43}$ Embajada española en Kabul: http://www.exteriores.gob.es/Embajadas/KABUL/es/VivirEn/Paginas/Seguridad. aspx [Consultado el 20 de abril de 2017]

44 Traducción de la autora. Embajada Gran Bretaña en Kabul: https://www.gov.uk/foreign-travel-advice/afghanistan [Consultado el 20 de abril de 2017]

45 Traducción de la autora. Embajada italiana en Kabul: http://www.ambkabul.esteri.it/ambasciata_kabul/en/ informazioni_e_servizi/informazioni-di-sicurezza.html [Consultado el20 de abril de2017]

${ }^{46}$ Traducción de la autora. Fuente, artículo escrito por Alice Févre la experta legal del Greek Forum of Refugees en Atenas "Not too late to act responsibly: stop deporting Afghan to un unsafe country!": http://refugees.gr/notlate-act-responsibly-stop-deporting-afghans-unsafe-country/ [Consultado el 20 de abril de 2017] 
ciudadanos afganos?"47

\section{Situación en Pakistán}

Pakistán es el paradigma de un estado frágil que experimenta más de seis décadas de inestabilidad política caracterizadas por falta de bienestar social, difícil acceso y distribución injusta de oportunidades (económicas, educativas y laborales), crecimiento del islamismo violento y sectario y violaciones frecuentes de Derechos Humanos que sufre la población civil como explica Zahid Hussain ${ }^{48}$. Su crisis no se centra únicamente en el estado, sino que, como apunta Raza Ahmad Rumi, hay una necesidad urgente de democratización del país y necesidad de un cambio de enfoque hacia la "seguridad humana" lejos de las nociones arcaicas de "seguridad nacional" que están ancladas en la militarización tanto de la sociedad como del estado ${ }^{49}$.

Alrededor del $60 \%$ de su territorio permanece ingobernable ${ }^{50}$ hecho que se traduce en inestabilidad en el país en las regiones de FATA, Baluchistán y el sur de Punyab, además de dificultades en el mantenimiento de la paz para con los países vecinos: Afganistán, India, Irán y países del Asia Central.

Human Rights Watch en su informe anual de 2016 sobre Pakistán, denunció la muerte de decenas de personas en bombardeos a mezquitas y juzgados como objetivos militares. La aplicación de ley, acciones policiales y las agencias de seguridad nacional siguen perpetrando violaciones de Derechos Humanos y ejerciendo una desproporcionada influencia política especialmente en asuntos de seguridad nacional y lucha contra el terrorismo.

Sigue vigente la pena de muerte y al menos 85 personas fueron condenadas a muerte y ejecutadas en 2016. A pesar de las denuncias por parte de la comunidad internacional y Naciones Unidas ${ }^{51}$, los tribunales militares secretos continúan operando y dictando sentencias de muerte.

Las mujeres, las minorías religiosas y las personas transgénero sufren ataques violentos, inseguridad y persecución. El gobierno continúa sin proporcionar una protección adecuada y tampoco pone los medios para investigar y responsabilizar a los perpetradores.

Informes de varias organizaciones internacionales denuncian las violaciones constantes de Derechos Humanos que tienen lugar en Pakistán en mayor medida contra minorías y colectivos vulnerables así como los ataques terroristas que se dan de manera asidua en el país 52.

47 Ibídem

${ }^{48}$ Zahid Hussain lo menciona en su artículo: "The construction and deconstruction of Pakistan: The institutional writ of the State".

${ }^{49}$ RUMI, Raza Ahmad, "Pakistan: Ungoverned Spaces", Telling the story, 2016, p. 179.

${ }^{50}$ Como describe Christian A. Carr en "Civil-Military Engagement Program: Enhancing the Mission of Regionally Engaged Army Forces".

${ }^{51}$ El relator especial sobre ejecuciones extrajudiciales, sumarias o arbitrarias Christof Heys lo denuncia en su informe de 2015 https://daccess-ods.un.org/TMP/5427633.52394104.html [Consultado el 21 de abril de 2017]

52 Amnistía Internacional informe anual sobre Pakistán: "Grupos armados continuaron perpetrando ataques dirigidos contra civiles, incluyendo empleados del gobierno, lo que resultó en cientos de bajas. Las fuerzas de 


\section{Conclusiones}

A través de la revisión de diferentes acuerdos y medidas tomadas por la UE podemos entender que hasta el momento, la mayoría de éstas no han sido lo suficientemente profundas como para abordar de forma sistémica la actual situación migratoria europea. Las respuestas políticas de la UE tanto a nivel interno (Agenda Europea para la Migración y redefinición del Sistema Europea Común de Asilo) como en cooperación con terceros países (Declaración UE-Turquía y acuerdos de retorno con Afganistán, son algunos de los ejemplos), siguen en desarrollo y presentan dificultades a la hora de hacer frente a la situación actual, quedándose cortas, desactualizadas y proponiendo soluciones lentas que no se corresponden con la idea de una Europa solidaria y respetuosa con los Derechos Humanos. En cambio, como apuntan Sergio Carrera, Steven Blockmans, Daniel Gros y Elisabeth Guild, se ha dado prioridad a la seguridad (asuntos de interior) y las preocupaciones e intereses militares de la UE y de sus estados miembros, poniendo el foco en los controles fronterizos, el retorno, la readmisión y la lucha contra el tráfico de personas en lugar de centrarse en asegurar el pleno cumplimiento de los Derechos Humanos fundamentales. Esto constituye uno de los talones de Aquiles de la actual Agenda sobre la Migración ${ }^{53}$.

A diferencia de lo que ocurre con el conflicto sirio, existe un gran desconocimiento sobre lo que ocurre en países como Afganistán o Pakistán. La situación de estos países pasa desapercibida en Europa, las presiones por parte de la sociedad civil y las organizaciones (aunque han ido en aumento) no son suficientes y no juegan un papel determinante en la política migratoria europea, que como hemos visto basan sus líneas de actuación en cuestiones de seguridad en vez de solidaridad. Todo esto deja el camino libre a la Comisión Europea para redactar programas y medidas especiales que perjudican a las personas que proviniendo de estos países llegan a Europa buscando paz, protección y seguridad.

De este modo, analizando los mecanismos de emergencia puestos en marcha por la UE para hacer frente a la situación migratoria actual y los programas especiales destinados a cubrir las necesidades básicas de las personas solicitantes de asilo, se pueden entender las relaciones que la UE establece con determinados países así como el acceso a programas que da a unas nacionalidades y otras deniega. Esto supone un claro ejercicio de discriminación dependiendo de la nacionalidad del solicitante de asilo. Así, unas personas disfrutan de un procedimiento más rápido a la hora de tramitarse su petición de asilo mientras otras experimentan difícil acceso o directamente son blanco de deportación.

Dependiendo de la nacionalidad de la persona solicitante de asilo, ésta tendrá acceso a unos programas de alojamiento especiales (refugios, apartamentos compartidos u hoteles

seguridad, particularmente los paramilitares Rangers en Karachi, cometieron violaciones de Derechos Humanos con casi total impunidad. Las ejecuciones continuaron, a menudo a consecuencia de juicios injustos. Los actores estatales y no estatales continúan discriminando a las minorías religiosas. A pesar de una nueva ley en Punyab para proteger a las mujeres de la violencia de género, los llamados crímenes de "honor" continuaron siendo denunciados. Los defensores de Derechos Humanos y trabajadores de los medios de comunicación sufrieron amenazas, hostigamientos y abusos por parte de las fuerzas de seguridad del Estado y grupos armados. Las minorías siguen enfrentándose a la discriminación al no tener acceso ni poder disfrutar de los derechos económicos y sociales que les corresponden. El acceso a una atención sanitaria de calidad, en particular para las mujeres pobres y rurales, sigue siendo limitado". Traducción de la autora. https://www.amnesty.org/en/ countries/asia-and-the-pacific/pakistan/ [Consultado el 21 de abril de 2017]

53 CARRERA, Sergio, et al, "The EU's Response to the Refugee Crisis: Taking Stock and Setting Policy Priorities", Ceps Essay, n²0, diciembre 2015. 
habilitados) o en su defecto quedará relegada a permanecer en los centros de recepción/ retención y experimentar condiciones de vida infrahumanas (violencia física y sexual, falta de agua caliente y calefacción, alojamiento en tiendas de campaña y comida en mal estado).

Igualmente ocurre con los programas de retorno voluntario, estos van dirigidos especialmente a nacionalidades con baja tasa de reconocimiento. La difusión de la información así como el acceso a los mismos parece llegar de manera más fácil y rápida a las nacionalidades en las que el programa pone el foco.

Como se ha comprobado, los diferentes estados miembros no recomiendan a sus nacionales viajar a Afganistán al igual que tampoco ofrecen recomendaciones muy positivas si se desea viajar a Pakistán o Argelia, entre otros países. Algunos de los nacionales originales de los países mencionados han encontrado la manera de llegar a Europa buscando protección. Para los ciudadanos europeos estos países no son seguros, sin embargo a ojos de Europa las y los refugiados/ciudadanos/personas de segunda e incluso tercera clase provenientes de estos países sí son merecedoras de vivir en ellos. De este modo se entiende quienes pueden disfrutar o tienen garantizado el derecho a la protección internacional y búsqueda de asilo y qué personas experimentan difícil acceso y medidas discriminatorias.

\section{Lista de acrónimos}

$\begin{array}{ll}\text { ACNUR } & \text { Alto Comisionados de las Naciones Unidas para los Refugiados } \\ \text { CE } & \text { Comisión Europea } \\ \text { EASO } & \text { European Asylum Support Office } \\ \text { OIM } & \text { Organización Internacional para las Migraciones } \\ \text { ONU } & \text { Organización de las Naciones Unidas } \\ \text { ONG } & \text { Organización no Gubernamental } \\ \text { RABIT } & \text { Rapid Border Intervention Teams } \\ \text { UE } & \text { Unión Europea } \\ \text { UNICEF } & \text { United Nations International Children's Emergency Fund } \\ \text { UNFPA } & \text { United Nations Population Fund }\end{array}$

\section{Bibliografía}

ACNUR, "Nota sobre la Carga y el Mérito de la Prueba en las Solicitudes de Asilo", 16 de diciembre de 1998: http://www.acnur.org/t3/fileadmin/Documentos/BDL/2002/1906.pdf [Consultado el 16 de abril de 2017]

ALCAIDE, Xavier, "Por qué la crisis de los refugiados no es una crisis de refugiados", Por la Paz/Peace in Progress, n029, noviembre 2016.

ALPES, Jill y TUNABOYLU, Sevda, "The EU-Turkey deal:what happens to people who return to Turkey?, febrero de 2017: http://postdeportation.org/2017/02/the-eu-turkey-deal-what-happens-topeople-who-return-to-turkey/

AMINISTÍA INTERNACIONAL, "EU-Turkey deal: Europe's year of shame", 2017: https://www.amnesty. org/en/latest/news/2017/03/the-eu-turkey-deal-europes-year-of-shame/ [Consultado el 16 de abril de 2017]

AMNISTÍA INTERNACIONAL, "Pakistan Annual report", 2017: https://www.amnesty.org/en/countries/ asia-and-the-pacific/pakistan/report-pakistan/ [Consultado el 21 de abril de 2017]

AMNISTÍA INTERNACIONAL, "Refugees. Human Rights have no borders", 1997.

CARR, Christian A., "Civil-Military Engagement Program: Enhancing the Mission of Regionally Engaged Army Forces", Military Review, vol. 96, no 2, 2016.

CARRERA, Sergio, et al, "The EU's Response to the Refugee Crisis: Taking Stock and Setting Policy Priorities", Ceps Essay, n²0, diciembre 2015. 
CONSTANT, Amelie F., ZIMMERMANN, Klaus F. "Towards a new European refugee policy that works", DICE Report, vol. 14, no 4, 2016.

Comunicación COM 240 final, de la Comisión al Parlamento Europeo, al Consejo, al Comité Económico y Social Europeo y al Comité de las Regiones sobre "Una agenda europea de migración", 2015: http://eur-lex.europa.eu/legal-content/ES/TXT/HTML/?uri=CELEX:52015DC0240\&from=EN [Consultado el 18 de abril 2017]

Comunicación COM 205 final, de la Comisión al Parlamento Europeo y al Consejo Europeo y al Consejo, de 2 de marzo, sobre "Third Progress Report on the Partnership Framework with third countries under the European Agenda on Migration", 2017: https://eeas.europa.eu/sites/eeas/files/ com_2017_205_f1_report_from_commission_en_v8_p1_880005_0.pdf [Consultado el 21 de abril 2017]

EUROPEAN UNION AGENCY FOR FUNDAMENTAL RIGHTS, "Opinion of the European Union Agency for Fundamental Rights on fundamental rights in the 'hotspots' set up in Greece and Italy", 2016: http://fra.europa.eu/en/opinion/2016/fra-opinion-fundamental-rights-hotspots-set-greeceand-italy [Consultado el 18 de abril de 2017]

EUROPEAN UNION, EXTERNAL ACTION, "Join Way Forward on migration issues between Afghanistan and EU", 2016: https://eeas.europa.eu/sites/eeas/files/eu_afghanistan_joint_way_forward_ on_migration_issues.pdf [Consultado el 20 de abril de 2017]

FARGUES, Philippe, DI BARTOLOMEO, Anna, "Drowned Europe", Brief Policy, 2015.

FURTAK, T. Florian, "The refugee crisis-A challenge for Europe and the world", Journal of civil \& legal sciences, vol. 5, Issue 1, 2015.

GREEK COUNCIL FOR REFUGEES, "Greece country report", Asylum Information Database, 2017: http:// www.asylumineurope.org/reports/country/greece [Consultado el 17 de abril de 2017]

MINISTERIO GRIEGO DEL INTERIOR, "Questions and Answers", 2016: http://asylo.gov.gr/en/wpcontent/uploads/2016/10/Qandanswers_ENG_OCT_V6.pdf [Consultado el 16 de abril de 2017]

GREEK FORUM OF REFUGEES, "Not too late to act responsibly: stop deporting Afghan to un unsafe country!", February 2017: http://refugees.gr/not-late-act-responsibly-stop-deporting-afghans-unsafe-country/

HATTON, Timothy J., "Refugees and Asylum seekers, the crisis in Europe and the future of policy", Economic Policy, vol. 32, no 91, 2017, ps. 447-496.

HUMAN RIGHTS WATCH, "World report 2017:Pakistan", 2017: https://www.hrw.org/world-report/2017/ country-chapters/pakistan [Consultado el 21 de abril de 2017]

MAJIDI, Nassim, "Afghan and Somali (post-) conflict migration to the EU", Forced Migration Review, no $51,2016$.

NACIONES UNIDAS, ASAMBLEA GENERAL, "Informe del Relator Especial sobre las ejecuciones extraoficiales, sumarias y arbitrarias Christof Heyns: El uso de la tecnología de la información y la comunicaciones para garantizar el derecho a la vida". A/HCR/29/37 (24 de abril de 2015).

NAIR, Sami, Refugiados: frente a la catástrofe humanitaria, una solución real, Crítica, Barcelona, 2016. ORGANIZACIÓN INTERNACIONAL PARA LAS MIGRACIONES, Press release: "Information event in the framework of the Programme: The Implementation of Assisted Voluntary Returns including Reintegration Measures": https://greece.iom.int/en/news/iom-information-event-frameworkprogramme-\%E2\%80\%9C-implementation-assisted-voluntary-returns-including [Consultado el 18 de abril de 2017]

PARK, Jeanne, "Europe's migration crisis", New York: Council of Foreign Relations, 2015, ps. 311-325.

PRIETO, Beatriz, "Los hotspots, un eslabón débil en la gestión de la crisis de los refugiados", Real Instituto Elcano, 2016: http://www.realinstitutoelcano.org/wps/portal/rielcano_es/contenido?WCM_ GLOBAL_CONTEXT=/elcano/elcano_es/zonas_es/demografia+y+poblacion/ari25-2016-prietohotspots-eslabon-debil-gestion-crisis-refugiados [Consultado el 21 de abril de 2017]

RITZEN, Jo, KAHANEC, Martin, "A Sustainable Immigration Policy for the EU", A Second Chance for Europe, Springer International Publishing, 2017, ps. 155-181.

RUMI, Raza Ahmad, "Pakistan: Ungoverned Spaces", Telling the story, 2016.

RUTTIG, Thomas, "Preamble: Afghans as a second-class refugees in parts of Europe", En CIDOB Monografías, Telling the story, sources of tension in Afghanistan \& Pakistan: A regional perspective (2011-2016), octubre 2016.

TSOURDI, Evangelia Lilian, DE BRUYCKER, Philippe, "EU asylum policy: In search of solidarity and access to protection", Brief Policy, 2015. 


\section{RELACIONES INTERNACIONALES}

Revista académica cuatrimestral de publicación electrónica Grupo de Estudios de Relaciones Internacionales (GERI) Universidad Autónoma de Madrid, España

www.relacionesinternacionales.info

ISSN 1699 - 3950

ff facebook.com/RelacionesInternacionales

twitter.com/RRInternacional 